\title{
Profissionais da saúde, que assistem pacientes com Acidente Vascular Cerebral, necessitam de informação especializada
}

\author{
Health Professionals who treat stroke patients need specialized \\ information.
}

Priscila Parochi Neves ${ }^{1}$, Sissy Veloso Fontes ${ }^{2}$, Márcia Maiumi Fukujima ${ }^{3}$, Sandro Luis de Andrade Matas $^{3}$, Gilmar Fernandes do Prado4.

\begin{abstract}
RESUMO
O Acidente Vascular Cerebral (AVC) é uma doença que pode ocasionar incapacidade funcional e levar ao óbito. Sendo assim, é necessária a intervenção de uma equipe multidisciplinar, com formação especializada, para o manuseio desses pacientes. O objetivo desse estudo foi realizar pesquisa de campo, com profissionais da área da saúde, (enfermeiro, fisioterapeuta, assistente social, médico, fonoaudiólogo, terapeuta-ocupacional, nutricionista e psicólogo), sobre as dificuldades, dúvidas e importância de orientações sobre os cuidados de pacientes com AVC. Foi aplicado questionário a 146 profissionais da saúde, que possuem experiência no atendimento desses pacientes, nas cidades de São Paulo, Santo André, São Bernardo do Campo e São Caetano do Sul. Observou-se, nesse estudo, que a maioria dos profissionais da área da saúde apresentam dificuldades (85\%), dúvidas (58\%), e consideram importante receber orientações (91\%), em relação aos cuidados de pacientes com AVC, através de materiais didáticos eletrônicos ou impressos, e cursos de extensão. A análise da pesquisa de campo realizada mostrou a importância e necessidade de informação/formação especializada para a maioria dos profissionais da saúde, da amostra estudada, que atendem pacientes com AVC.
\end{abstract}

Unitermos: Acidente vascular cerebral, Educação continuada, Profissionais da saúde, Cuidados especializados.

\section{SUMMARY}

Stroke can generate functional disability and death. It is necessary an intervention of a multidisciplinary team with specialized formation to handle those patients. The objective of this study was to accomplish field research with professionals of health area (nursing, physiotherapy, social work, physician, speech therapy, occupational therapy, nutritionist and psychologist) on the difficulties, doubts, and importance of orientations on caring of patients with stroke. 146 questionnaires were applied to health professionals,

Trabalho realizado:Universidade Metodista de São Paulo -UMESP

1- Fisioterapeuta, Especialista em Fisioterapia Neurológica pela UMESP

2- Fisioterapeuta, Professora de Educação Física, Mestre em Neurociências e Doutora em Ciências pela UNIFESP, Docente da UMESP e UNISANTA

3- Neurologista da UNIFESP

4- Neurologista e Docente da UNIFESP

Endereço para correspondência: Sissy Veloso Fontes

R: Francisco Tapajós, 513 apto. 122 - Jd. Santo Estéfano São Paulo - SP - CEP: 04153001

Tel. (1 1) 50585105 - email: sissyfontes@sti.com.br

Trabalho recebido em 20/10/04. Aprovado em 23/11/04 
with experience on treatment of those patients, in Sao Paulo, Santo André, Sao Bernardo do Campo and Sao Caetano do Sul. It was observed, in this study, that most health professionals has difficulties (85\%), doubts (58\%), and they consider important to receive orientations $(91 \%)$ regarding care of patients with stroke, through electronic or printed didactic materials, and extension courses. This research showed the importance and need of specialized information / formation for most of the health professionals on our sample that assist patients with stroke.

Keywords: Stroke, Continued education, Health professionals, Specialized care.

\section{INTRODUÇÃO}

$O$ acidente vascular cerebral (AVC) é a doença que mais ocasiona mortes em nosso país ${ }^{1,2}$ e que mais incapacita no mundo ${ }^{3}$. Esta doença pode ocasionar alterações cognitivas e neuromusculares, levando a problemas psicoemocionais e sócio-econômicos ${ }^{4}$. Sendo assim, a atuação de diversos profissionais da área da saúde é imprescindível para assistência adequada e integral nestes casos ${ }^{5}$.

A busca das melhores evidências disponíveis, integrada à experiência clínica, tem sido sugerida como a estratégia ideal e mais segura para a tomada de decisão em saúde. Esta conduta é considerada prática baseada em evidências ${ }^{6}$. Para uma assistência favorável, por parte dos profissionais que atendem pacientes com AVC, esta prática possibilita um manejo especializado com base em conhecimentos científicos específicos, propiciando melhores resultados terapêuticos ${ }^{7,8}$. No entanto, há indícios de que esta prática não tem sido utilizada na rotina clínica de muitos profissionais da saúde que prestam cuidados às vítimas de AVC, apesar de haverem várias diretrizes (guidelines) publicadas na literatura nacional e internacional.

Além da utilização de informações científicas para guiar a prática clínica, tem-se inferido que cursos de educação continuada nas áreas da saúde fornecem vias promissoras de ensino para a prática clínica especializada9 .

Investigar se existe rotina quanto à utilização de diretrizes para guiar a prática clínica, identificar as dificuldades e dúvidas no manejo de pacientes com AVC pelos profissionais da área da saúde que atuam em instituições na cidade de São Paulo e região do $A B C$ e colher opiniões quanto ao interesse em receber informações e formação especializada para atuação profissional, nestes casos, são os objetivos deste trabalho.

\section{MÉTODO}

Foi aplicado questionário aos profissionais: enfermeiro, médico, nutricionista, fisioterapeuta, terapeuta ocupacional, fonoaudiólogo, psicólogo e assistente por um mesmo entrevistador nos meses de julho a outubro de 2002. Todos os profissionais entrevistados, deveriam ter alguma experiência quanto aos cuidados de pacientes com AVC e pertencer a uma das seguintes instituições da cidade de São Paulo e região do ABC: Centro de Reabilitação Lar Escola São Francisco (LESF), Divisão de Medicina de Reabilitação (DMR) - Universidade de São Paulo, Clínica de Fisioterapia da Universidade Bandeirante de São Paulo - Campus ABC, Clínica Escola de Fisioterapia da Universidade Metodista de São Paulo, Hospital São Caetano, Hospital Santa Cruz, Hospital Santa Casa de Santo André, Hospital Santa Marta, Hospital Municipal de Itapecerica da Serra e Hospital São Paulo Universidade Federal de São Paulo.

O questionário aplicado consta de 3 domínios: 1 - Dados Pessoais (nome, idade, sexo, escolaridade, profissão, local e perfil econômico do serviço que trabalha); 2 - Acidente Vascular Cerebral (principais dificuldades e dúvidas em relação aos cuidados de pacientes com AVC); 3 Orientações (Já recebeu alguma orientação profissional sobre a sua conduta específica no manejo do AVC? Que profissionais? Utiliza ou já utilizou alguma diretriz, guia ou manual sistematizado de orientações profissionais? Considera importante receber orientações profissionais para a sua prática? Gostaria de receber orientações profissionais quanto às múltiplas áreas de atuação? Em quais aspectos? Como gostaria de receber orientação, em cursos de educação continuada, por material didático (eletrônico ou impresso) ou ambos? Você pode sugerir algumas orientações específicas de sua 
área de atuação a serem ministradas aos profissionais que assistem pacientes com AVC? Se, sim quais?). Este foi aplicado por meio de entrevista aos diversos profissionais nos próprios locais de trabalho, que concordaram em participar do estudo e aprovaram a utilização dos resultados para fins de pesquisa, desde que houvesse sigilo quanto à identificação dos entrevistados.

Os resultados quantitativos são apresentados em percentual e os qualitativos descritivamente.

\section{RESULTADOS}

Foram entrevistados 146 profissionais da área da saúde, sendo 34 (26\%) enfermeiros (enf), 33 (22\%) fisioterapeutas (ft), 27 (18\%) assistentes sociais (ass), 25 (17\%) médicos neurologistas e fisiatras (md), 10 (7\%) fonoaudiólogos (fo), 8 (5\%) terapeutas ocupacionais (to), 5 (3\%) nutricionistas (nu) e 4 (2\%) psicólogos (psc).

\section{Características Demográficas}

A média das idades dos entrevistados foi de 35 anos, sendo 29\% do sexo masculino.

Em relação ao tempo de formação profissional $32 \%$ possuem mais de 10 anos, 38\% estão entre 5 a 10 anos e, 30\% a menos de 5 anos. Mais da metade dos profissionais, $53 \%$ eram pósgraduados (37\% especialistas, 13\% mestres, 3\% doutores).

Quanto à via de recebimento de honorários dos serviços em que trabalham, $43 \%$ recebem do sistema único de saúde (SUS), 7\% de convênios, $10 \%$ de particulares e $40 \%$ por via mista (SUS + convênios + particulares).

\section{Dados Quantitativos}

Estão apresentados nas tabelas 1, 2, 3, 4, 5, 6, 7 e 8.

\section{Dados Qualitativos}

\section{A) Dificuldades}

Quanto às dificuldades encontradas, os enfermeiros citaram aspectos como posicionamentos e transferências adequadas, aspectos relacionados à imobilidade, dependência funcional e diminuição do nível de consciência, prevenção e tratamento de úlceras por pressão, cuidados na incontinência vesical, além de conhecimentos sobre processo e tempo de recuperação funcional, sinais preditores prévios ao episódio de AVC, cuidados com alimentação e tipo de dieta adequada, e como orientar familiares e cuidadores para prestarem assistência ao paciente.

Tanto os fisioterapeutas como os terapeutas ocupacionais relataram dificuldades em lidar com sintomas clínicos inerentes a algumas síndromes neurológicas específicas como, por exemplo, nas alterações de linguagem (afasias), psicológicas (depressão e ansiedade), situação sócioeconômica baixa e falta de colaboração por parte do familiar e ou cuidador no programa terapêutico. Outro aspecto, também mencionado, foi à falta de interação entre os profissionais, como já descrito por alguns autores ${ }^{19}$.

De acordo com os assistentes sociais entrevistados, mais da metade relataram dificuldades em prestar assistência a pacientes com alterações emocionais e de comunicação, como também em reconhecer os preditores prognósticos do AVC e, como poderiam orientar melhor a família e o paciente em relação aos aspectos específicos de sua área de atuação.

Os médicos apresentaram dificuldades em caracterizar a história da moléstia atual do paciente, quando contada por familiares e ou

\begin{tabular}{|c|c|c|c|c|c|c|c|c|c|c|c|c|c|c|c|c|c|c|}
\hline$r$ & \multicolumn{2}{|c|}{ enf } & \multicolumn{2}{|l|}{ pt } & \multicolumn{2}{|c|}{ ass } & \multicolumn{2}{|c|}{ md } & \multicolumn{2}{|l|}{ fo } & \multicolumn{2}{|l|}{ to } & \multicolumn{2}{|c|}{ nu } & \multicolumn{2}{|c|}{ psc } & \multicolumn{2}{|c|}{ Total } \\
\hline Dif & $\mathrm{n}$ & $\%$ & $\mathbf{n}$ & \begin{tabular}{|l}
70 \\
\end{tabular} & $n$ & $\%$ & $n$ & $\%$ & $\mathbf{n}$ & $\%$ & $\mathrm{n}$ & $\%$ & $\mathbf{n}$ & $\%$ & $n$ & $\%$ & & $\%$ \\
\hline & 2 & 85 & 3 & 04 & & 78 & & 7 & & 9 & 7 & 87 & & 10 & & 75 & 24 & 8 \\
\hline & & 15 & 2 & 6 & 6 & 22 & 0 & 24 & & 10 & 1 & 13 & & 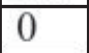 & & 25 & 22 & \\
\hline
\end{tabular}

Tabela 1. Profissionais da saúde segundo dificuldades ao prestar assistência para paciente com AVC.

\begin{tabular}{|c|c|c|c|c|c|c|c|c|c|c|c|c|c|c|c|c|c|c|}
\hline & \multicolumn{2}{|c|}{ enf } & \multicolumn{2}{|l|}{ pt } & \multicolumn{2}{|c|}{ ass } & \multicolumn{2}{|c|}{ md } & \multicolumn{2}{|l|}{ fo } & \multicolumn{2}{|l|}{ to } & \multicolumn{2}{|c|}{ nu } & \multicolumn{2}{|c|}{ pse } & \multicolumn{2}{|c|}{ Total } \\
\hline & $n$ & $\%$ & 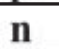 & $\%$ & $n$ & $\%$ & $\mathrm{~N}$ & $\%$ & $\mathbf{n}$ & 170 & $n$ & $\%$ & 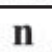 & $\%$ & $n$ & $\%$ & n & $\%$ \\
\hline & & & & & & & & & 5 & & & & & & & & & 58 \\
\hline & 14 & 41 & 12 & 36 & 11 & 41 & 13 & 52 & \begin{tabular}{|l|}
5 \\
\end{tabular} & 50 & 2 & 25 & & 20 & & 100 & 62 & $4 ?$ \\
\hline
\end{tabular}

Tabela 2. Profissionais da saúde segundo dúvidas quanto à sua conduta na assistência ao paciente com AVC. 


\begin{tabular}{|c|c|c|c|c|c|c|c|c|c|c|c|c|c|c|c|c|c|c|}
\hline Pro & enf & & pt & & ass & & md & & fo & & to & & $\mathrm{nu}$ & & ps & & Tot & \\
\hline Ori & $\mathbf{n}$ & $\%$ & n & $\%$ & $\mathbf{n}$ & $\%$ & n & $\%$ & $\mathrm{n}$ & $\%$ & $\mathbf{n}$ & $\%$ & $\mathbf{n}$ & $\%$ & n & $\%$ & $\mathrm{n}$ & 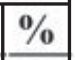 \\
\hline- & 21 & 6 & - & 91 & $\sigma$ & $=$ & 1 & 56 & 10 & 100 & 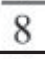 & 100 & 3 & 60 & & 100 & 96 & 66 \\
\hline ao & 13 & 39 & 2 & 9 & 21 & 78 & 11 & 44 & 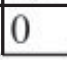 & 0 & 0 & 0 & 2 & 40 & 0 & 0 & 50 & 13 \\
\hline
\end{tabular}

Tabela 3. Profissionais da saúde segundo as orientações recebidas por profissionais da saúde em relação à assistência ao paciente com AVC.

\begin{tabular}{|c|c|c|c|c|c|c|c|c|c|c|c|c|c|c|c|c|c|c|}
\hline Profissionais & enf & & pt & & ass & & md & & fo & & to & & nu & & ps & & Tota & \\
\hline Guia & $\mathbf{n}$ & $\%$ & $\mathbf{N}$ & $\%$ & $\mathbf{n}$ & $\%$ & $\mathrm{n}$ & $\%$ & $\mathbf{n}$ & $\%$ & $\mathbf{n}$ & $\%$ & $\mathbf{n}$ & $\%$ & $\mathbf{n}$ & $\%$ & $\mathbf{n}$ & $\%$ \\
\hline Sim & 5 & 15 & 5 & 15 & 1 & 3 & 3 & 12 & 5 & 50 & 1 & 12 & 2 & 40 & 2 & $\overline{50}$ & 24 & 16 \\
\hline Não & 29 & 85 & 28 & 85 & 26 & 97 & 22 & 88 & 5 & 50 & 7 & 88 & 3 & 60 & 2 & 50 & 122 & 84 \\
\hline
\end{tabular}

Tabela 4. Profissionais da saúde segundo a utilização de diretrizes, guia ou manual de orientações profissionais para a assistência ao paciente com AVC.

\begin{tabular}{|c|c|c|c|c|c|c|c|c|c|c|c|c|c|c|c|c|c|c|}
\hline Pro & \multicolumn{2}{|c|}{ enf } & \multicolumn{2}{|l|}{ pt } & \multicolumn{2}{|c|}{ ass } & \multicolumn{2}{|c|}{ md } & \multicolumn{2}{|l|}{ fo } & \multicolumn{2}{|c|}{ to } & \multicolumn{2}{|c|}{ nu } & \multicolumn{2}{|c|}{ pse } & \multicolumn{2}{|c|}{ Total } \\
\hline Im & $\mathrm{n}$ & $\%$ & n & $\%$ & $\mathbf{N}$ & $\%$ & $\mathbf{N}$ & $\%$ & n & $\%$ & $n$ & $\%$ & $\mathrm{n}$ & $\%$ & $n$ & $\%$ & - & $\%$ \\
\hline & 3 & 95 & 3 & 90 & 26 & 9 & & 3 & & 90 & 6 & 7 & & 8 & 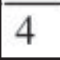 & 10 & 03 & 91 \\
\hline & & 5 & 3 & 10 & 1 & 4 & 3 & 12 & & 10 & 2 & 25 & & 20 & 0 & 0 & 13 & 9 \\
\hline
\end{tabular}

Tabela 5. Profissionais da saúde segundo a importância em receber orientações profissionais para a assistência ao paciente com AVC.

\begin{tabular}{|c|c|c|c|c|c|c|c|c|c|c|c|c|c|c|c|c|c|c|}
\hline Profissionais & enf & & pt & & ass & & md & & fo & & to & & $\mathrm{nu}$ & & ps & & Tota & \\
\hline Gostaria & n & $\%$ & n & $\%$ & $\mathbf{n}$ & $\%$ & $\mathbf{n}$ & $\%$ & $\mathbf{n}$ & $\%$ & $n$ & $\%$ & $\mathbf{n}$ & $\%$ & n & $\%$ & $\mathbf{n}$ & $\%$ \\
\hline Sim & 32 & 94 & 27 & 82 & 25 & 93 & 21 & 84 & 7 & $\overline{70}$ & 6 & 75 & 4 & 80 & 2 & 50 & 124 & 85 \\
\hline Não & 2 & 6 & 6 & 18 & 2 & 7 & 4 & 16 & 3 & 30 & 2 & 25 & 1 & 20 & 2 & 50 & 22 & 15 \\
\hline
\end{tabular}

Tabela 6. Profissionais da saúde que gostariam de receber orientações profissionais para a assistência ao paciente com AVC nas diversas áreas de atuação.

\begin{tabular}{|c|c|c|c|c|c|c|c|c|c|c|c|c|c|c|c|c|c|c|}
\hline Profissionais & \multicolumn{2}{|c|}{ enf } & \multicolumn{2}{|l|}{ pt } & \multicolumn{2}{|l|}{ ass } & \multicolumn{2}{|c|}{ md } & \multicolumn{2}{|l|}{ fo } & \multicolumn{2}{|l|}{ to } & \multicolumn{2}{|c|}{ nu } & \multicolumn{2}{|c|}{ psc } & \multicolumn{2}{|c|}{ Total } \\
\hline Estratégia & $\mathbf{n}$ & $\%$ & $\mathbf{n}$ & $\%$ & $\mathbf{n}$ & $\%$ & $\mathbf{n}$ & $\%$ & $\mathbf{N}$ & $\%$ & $\mathbf{n}$ & $\%$ & $\mathbf{n}$ & $\%$ & $\mathbf{n}$ & $\%$ & $\mathbf{n}$ & $\%$ \\
\hline Cursos & 10 & 30 & 11 & 33 & 2 & 7 & 9 & 36 & 1 & 1 & 3 & 38 & 3 & 60 & 2 & 50 & 41 & 28 \\
\hline Material & 9 & 26 & 12 & 37 & 9 & 33 & 10 & 40 & 2 & 20 & 1 & 12 & 1 & 20 & 1 & 25 & 45 & 30 \\
\hline Ambos & 15 & 44 & 10 & 30 & 16 & 60 & 6 & 24 & 7 & 70 & 4 & 50 & 1 & 20 & 1 & 25 & 60 & 42 \\
\hline
\end{tabular}

Tabela 7. Profissionais da saúde segundo a preferência quanto à estratégia a ser orientada (em cursos de extensão, material didático - eletrônico ou impresso, ou ambos) quanto à assistência ao paciente com AVC pelos diversos profissionais.

\begin{tabular}{|c|c|c|c|c|c|c|c|c|c|c|c|c|c|c|c|c|c|c|}
\hline Profissionais & enf & & pt & & ass & & md & & fo & & to & & nu & & psc & & Tot & \\
\hline Sugestão & n & $\%$ & n & $\%$ & $\mathbf{n}$ & $\%$ & n & $\%$ & $n$ & $\%$ & $n$ & $\%$ & n & $\%$ & n & $\%$ & $\mathbf{n}$ & $\%$ \\
\hline Sim & 14 & $\overline{41}$ & 12 & 36 & 5 & 19 & 6 & 24 & 6 & 60 & 3 & 37 & 2 & 40 & 2 & 50 & 50 & 35 \\
\hline Não & 20 & 59 & 21 & 64 & 22 & 81 & 19 & 76 & 4 & 40 & 5 & 63 & 3 & 60 & 2 & 50 & 96 & 65 \\
\hline
\end{tabular}

Tabela 8. Profissionais da saúde que sugeriram orientações, quanto à sua atuação profissional para os diversos profissionais que assistem o paciente com AVC. 
cuidadores, interpretar os sintomas, eleger as melhores estratégias de tratamento e, atender com qualidade em um curto período de tempo em serviços da saúde que apresenta grande demanda e número insuficiente de profissionais médicos. Além disso, citaram dificuldades quanto a sua intervenção, quando o paciente apresenta alterações psicológicas, de linguagem, dificuldades financeiras, e seqüelas sensório-motoras. Avaliar as disfunções deglutitórias, tratar complicações clínicas, principalmente as infecções, abordar a relação paciente-familiar e, obter aderência ao tratamento prescrito, também foram dificuldades citadas.

Já os fonoaudiólogos apresentaram dificuldades quanto ao posicionamento correto do paciente, para uma melhor emissão da linguagem e para a alimentação, como abordar pacientes com alteração psicológica, como orientar a família para colaborar com o programa proposto, quais as melhores estratégias a serem utilizadas no tratamento das disfagias. Além disso, também relataram dificuldade na relação interdisciplinar.

Os nutricionistas apresentaram dificuldades relacionadas às outras áreas da saúde, por exemplo: como abordar pacientes com alteração de linguagem, como alimentar e orientar os cuidadores, como orientar a família na mobilização adequada desses pacientes.

Os psicólogos relataram dificuldades quanto à intervenção em pacientes com déficit de linguagem, memória, nível sócio-econômico precário e a falta de colaboração da família no processo terapêutico.

\section{B) Dúvidas}

Em relação às dúvidas dos profissionais da saúde sobre os cuidados dos pacientes com AVC, observamos que apenas os psicólogos não as apresentaram, os demais, mais da metade apresentaram.

As dúvidas citadas pelos enfermeiros foram, principalmente em relação à medicação, alimentação, mudança de decúbito, órteses, cuidados com a bexiga neurogênica, orientações quanto à sexualidade e, como poderiam intervir, favoravelmente na melhora da auto-estima de muitos pacientes psicologicamente abalados. Como orientar a família quanto aos cuidados domiciliares e como identificar precocemente os sinais e sintomas do início de um novo icto, também foram citadas.
Os fisioterapeutas apresentaram dúvidas, sobre: os efeitos no sistema neuromuscular das medicações prescritas nos casos de AVC, indicação de adaptações (órteses) ideais em determinados casos, como poderiam colaborar, enquanto fisioterapeutas na melhora das disfunções de linguagem e de deglutição, cuidados com a traqueostomia, como melhor orientar sobre a realização das atividades de vida diária (AVD's) e de vida prática (AVP's) no ambiente real dos pacientes, e sobre o tempo estimado para a recuperação sensório-motora. As terapeutas-ocupacionais citaram dúvidas semelhantes aos dos fisioterapeutas, como as relacionadas às adaptações, orientações quanto à realização correta de AVD's e AVP's utilizando o hemicorpo acometido, além de condutas nas alterações cognitivas e, quanto à atuação dos demais profissionais que assistem o paciente com AVC.

As respostas dos assistentes sociais, em relação as suas dúvidas foram: sobre o tratamento do AVC em geral, como se relacionar com o paciente e o familiar, como transferir pacientes gravemente acometidos, as limitações físicas advindas desta doença, quanto às possíveis intercorrências clínicas, quando devem começar a fisioterapia e para que serviços encaminhar, aspectos sobre a epidemiologia da doença e questões previdenciárias.

As dúvidas indicadas pelos médicos foram sobre: qual seria a melhor conduta clínica a ser tomada, quando utilizar fibrinolíticos, como poderiam intervir nas alterações de linguagem e o prognóstico destas, a eficácia do tratamento fonoaudiológico, e fisioterapêutico, o que deveriam indicar de exercícios físicos, prognóstico sensóriomotor, questões sobre o transporte público gratuito e, se os pacientes seguem devidamente as orientações por eles ministradas.

Já as fonoaudiólogas apresentaram dúvidas sobre o prognóstico funcional, tratamento médico, prevenção de úlceras por pressão, sondas para alimentação e cuidados com a traqueostomia, principalmente.

As nutricionistas, também relataram possuir dúvidas em relação ao prognóstico funcional. As dúvidas quanto à identificação do nível de compreensão do paciente e do tipo de disfagia, de acordo com a área da lesão, foram citadas. 


\section{C) Orientações Recebidas}

Quanto às orientações recebidas, os enfermeiros foram orientados por médicos sobre os medicamentos e sobre aspectos inerentes ao AVC, por fisioterapeutas sobre como mobilizar os pacientes, por terapeutas ocupacionais sobre as AVD'S, por fonoaudiólogos sobre os cuidados com a deglutição e, por outros enfermeiros sobre cuidados de higiene.

Os fisioterapeutas receberam orientações de médicos e dos próprios colegas de profissão, seguido de fonoaudiólogos, terapeutasocupacionais, assistente-social, enfermagem, nutricionista e psicólogo. Estas foram, respectivamente sobre medicamentos prescritos, evolução clínica, posicionamento, transferências e AVD'S adequados, cuidados com a deglutição, prescrição de órteses, quanto aos responsáveis em auxiliar nos tramites da aposentadoria e do transporte gratuito, prevenção e cuidados com úlceras por pressão, sobre o uso de sondas nasoenteral e vesical e, incontinência urinária, alimentos com contra indicação relativas ou absolutas em casos clínicos especiais, quanto à identificação e encaminhamento de pacientes deprimidos e ou ansiosos aos psicólogos.

De acordo com os dados obtidos, todos os terapeutas-ocupacionais já receberam orientações dos próprios terapeutas-ocupacionais sobre as AVD'S, de fisioterapeutas sobre posicionamentos, exercícios de alongamento e manuseios em geral, dos fonoaudiólogos sobre a comunicação e a deglutição, dos médicos sobre o tratamento medicamentoso e ou cirúrgico e de psicólogos sobre as alterações emocionais dos pacientes com AVC.

As orientações recebidas pelos assistentes sociais foram dadas por médicos no que se referia a diagnóstico, prognóstico e tratamento clínico, enfermagem quanto ao uso de sondas, terapeutaocupacional e fisioterapeuta quanto aos posicionamentos.

Mais da metade dos médicos receberam orientações, principalmente de outros médicos (sobre hipóteses do diagnóstico clínico, análise dos exames complementares como a tomografia computadorizada de crânio, tratamento clínico e ou cirúrgico e sobre os processos de recuperação física), seguido de fisioterapeutas sobre os efeitos dos exercícios físicos nesta população e, de fonoaudiólogos sobre a avaliação e tratamento de distúrbios da deglutição e de linguagem.

Em relação aos fonoaudiólogos todos, já receberam orientações dos demais profissionais sobre posicionamentos e posturas mais adequadas para alimentar os pacientes ou orientálos para tal, a melhor dieta alimentar, o estado emocional, bem como sobre a evolução clínica destes.

Os nutricionistas (mais da metade) já receberam, de toda a equipe, principalmente fisioterapeuta, fonoaudiólogo e enfermeiro, orientações sobre posicionamentos e mobilização do paciente, dieta e cuidados gerais.

Já os psicólogos foram unânimes em relatar terem sido orientados por médicos e fisioterapeutas especialmente, e depois por terapeuta-ocupacional, fonoaudiólogo e psicólogo sobre diagnósticos, intervenções terapêuticas e manejo destes pacientes.

\section{D) Interesse em Receber Orientações}

$\mathrm{Na}$ questão sobre o interesse em receber orientações, os enfermeiros citaram aspectos sobre o diagnóstico clínico, aspectos preventivos e curativos da doença, do exame físico, do quadro clínico, de mecanismos relacionados à deglutição, à comunicação, à sexualidade e à recuperação funcional dos pacientes, quanto aos cuidados com a pele, sondas, posicionamentos e manuseios, e outros procedimentos gerais da enfermagem.

Mais da metade dos fisioterapeutas gostaria de ser orientado por todos os demais profissionais, principalmente das áreas de psicologia e de fonoaudiologia, corroborando os resultados deste estudo no concernente às maiores dificuldades citadas por estes profissionais. Os terapeutasocupacionais relataram a importância de receber orientações de outras áreas correlatas quanto aos manuseios, exercícios físicos terapêuticos, quanto a outras adaptações das AVD'S, e quanto a estratégias de estimulação da linguagem.

As orientações preferidas pelos assistentes sociais constam aspectos sobre diagnóstico, etiologia, prevenção, quadro clínico, recuperação e seqüelas do AVC, função do serviço social, relação terapeuta-paciente e familiar, enquanto que os médicos preferem orientações quanto às estratégias que propiciam a recuperação motora, e sobre as intervenções psicológicas, sociais e clínicas atuais.

Fonoaudiólogos gostariam de ser orientados sobre a mobilização dos indivíduos e como prognosticar a evolução motora, e os nutricionistas gostariam de receber informações sobre o processo geral de recuperação, em especial estratégias de como se deve alimentar estes pacientes, em especial os disfágicos, enquanto que os psicólogos sobre os diversos diagnósticos e manejos do paciente. 
E) Orientações que os vários profissionais ministraram, sugeriram e aspectos que esclareceram

- Enfermagem: o enfermeiro sugere e ministra informações sobre alimentação, hidratação, higiene, mudança de decúbitos, prevenção de escaras, massagem, orientações quanto à autoajuda para executar as AVD's. Relatam serem estes, os principais aspectos dos cuidados de enfermagem. Defendem que educar e esclarecer os pacientes, bem como seus familiares quanto aos cuidados gerais de saúde são quesitos básicos da atuação desta área.

- Fisioterapia: o fisioterapeuta sugere e ministra informações sobre prevenção e intervenção nas complicações clínicas, recuperação motora funcional (por meio de posicionamentos, exercícios físicos terapêuticos facilitadores da execução voluntária das AVD's e AVP's ministrados e orientados), mediar processos de inclusão social, orientar, incentivar e encaminhar para prática esportiva, de lazer e profissional. O principal objetivo desta área é estimular funções neuromotoras, procurando integrar aspectos físicos com os psicológicos e sociais, aspectos estes inerentes e indissociáveis a todos os indivíduos.

- Terapia ocupacional: o terapeuta ocupacional ministra e sugere informações sobre AVD's, AVP's, orientações aos familiares e ou cuidadores.

- Ação da assistente social: o assistente social ministra e sugere informações sobre orientações sobre a relevância do encaminhamento do paciente com AVC para o serviço social, atenção especial para a interação entre os profissionais técnicos que prestam assistência a estes indivíduos, com o paciente e seus familiares visando à integração social (relações interpessoais) do grupo em questão.

- Intervenção médica: o médico sugere e ministra informações sobre prevenção primária e secundária (orientação e tratamento), diagnóstico clínico, terapêutica medicamentosa e, quando necessária cirúrgica. Afirmam que a eficácia do tratamento está diretamente relacionada ao reconhecimento dos sinais e sintomas da doença e à intervenção adequada. Enfatizam a importância dos cuidados com a pressão arterial sistêmica e reconhecem a relevância de intervenção terapêutica dos demais profissionais da saúde.
- Fonoaudiologia: os fonoaudiólogos ministram e sugerem informações sobre deglutição, comunicação (tratamento e técnicas alternativas).

- Nutrição: o nutricionista sugere e ministra informações sobre análise e composição de dietas alimentares. Relatam ser, a orientação, primordial para uma melhor qualidade de atendimento aos pacientes e familiares.

- Psicologia: o psicólogo sugere e ministra informações sobre funções neuropsicológicas e afetivo-emocionais e realização de dinâmicas familiar.

\section{DISCUSSÃO}

Mais da metade dos profissionais são formados a mais de cinco anos e são pós-graduados. No entanto, o fato de serem pós-graduados e terem conhecimentos em áreas específicas da ciência, não mostrou ter sido suficiente para suprirem as dúvidas e dificuldades encontradas na assistência aos pacientes com AVC, pois quase a totalidade dos profissionais apresentou as mais diversas dificuldades, o que pode sugerir a necessidade de formação, por exemplo, ministrada em cursos de educação continuada, e de informação especializada para atuação profissional nesta área, como descrita em diversas diretrizes (guidelines) disponíveis na literatura ${ }^{5,7,8,10-19}$.

Muitos destes profissionais relataram apresentar, várias vezes ansiosa expectativa quanto à recuperação funcional dos seus pacientes, talvez porque estes, tenham percebido a forte relação existente entre o nível de incapacidade funcional e as alterações psicológicas (depressão e ansiedade), ou seja, quanto maior a incapacidade funcional mais deprimido o paciente pode estar. Este fato foi descrito por Carson e cols. em 2000, que relatam significante relação entre esses dois aspectos ${ }^{20}$.

Observou-se que muitos dos profissionais entrevistados relataram apresentar dificuldades e dúvidas relacionadas às atividades de suas próprias áreas de atuação, o que vem a corroborar com a afirmativa quanto à necessidade de formação e informação especializada para os diferentes profissionais nesta área de atuação neurológica específica. Um dos fatores que chamou a atenção são as dúvidas, que alguns profissionais possuem, em relação à orientação 
sobre aspectos não relacionados as suas áreas específicas de atuação, mostrando a dificuldade que estes possuem em identificar ou respeitar o papel específico de cada um dos profissionais da saúde que trabalham em uma equipe multidisciplinar. Alguns autores descrevem esta dificuldade como inerente das equipes multiprofissionais ${ }^{21}$. Os resultados descritivos deste estudo confirmam esta afirmativa, já que muitos dos profissionais parecem ignorar ou não aceitar as particularidades de atuação dos demais colegas profissionais.

Os assistentes sociais foram os únicos a terem sido, em minoria, orientados por seus colegas de profissão ou, das demais profissões. Em relação aos profissionais médicos, apenas a metade teve a oportunidade de ser orientado pelos diversos profissionais. Os demais profissionais foram, ou em sua totalidade ou na grande maioria orientados, o que provavelmente os favorece a compreender as diversas interfaces que esta doença pode acarretar, além de poder facilitá-los a compreender a relevância em trabalhar em equipes inter e não multidisciplinares.

Um dos dados mais relevante deste estudo foi a comprovação de que a utilização de diretrizes sobre atuação profissional nos cuidados de pacientes com AVC e a busca das melhores evidências científicas quanto à tomada de decisões clínicas não é rotina para a maioria dos profissionais entrevistados. A ausência desta rotina talvez advenha da falta de conhecimento, por parte de muitos destes profissionais, sobre a existência de várias diretrizes publicadas e disponíveis gratuitamente em bases de dados da literatura nacional e internacional ou, por não considerarem importante esta prática para suas atuações profissionais, podendo gerar condutas ineficientes e ou inapropriadas.

A minoria cita a utilização de alguns manuais, apostilas ou guias não sistematizados para orientar suas condutas, podendo, muitas vezes confrontar e divergir das informações científicas de maior validade disponíveis na literatura.

A visão, quase unânime dos diferentes profissionais sobre a importância em receber orientações, quanto à assistência ao paciente com AVC mostrou a preocupação destes, com o aperfeiçoamento do atendimento nesta área. De acordo com a maioria dos profissionais é importante receber novas orientações, trocar experiências profissionais, esclarecer dúvidas quanto aos conhecimentos pré-existentes utilizados na prática e estar atualizado quanto às condutas atuais mais eficientes.

A maioria dos profissionais gostaria de receber orientações quanto aos cuidados de pacientes com AVC, mostrando elevado grau de interesse, independente dos locais ou condições em que exercem sua profissão atualmente.

Em relação à preferência dos profissionais quanto à estratégia a ser utilizada para receberem orientações foi significativa à preferência por cursos, associado a materiais didáticos eletrônicos ou impressos, o que pode sugerir a necessidade de elaboração e aplicação de cursos de educação continuada com material previamente preparado para profissionais que atuam nos cuidados de pacientes com AVC nestas regiões do estado de São Paulo.

Todos os diferentes tipos de profissionais sugeriram orientações quanto a sua atuação profissional mas, menos da metade dos entrevistados responderam o que deveria ser orientado. Talvez estes não sabem como ou o que orientar, não se consideram aptos para orientar, ou simplesmente não tiveram interesse em colaborar com este quesito do questionário.

Podemos inferir que a medida que o profissional obtém esclarecimentos sobre outras áreas de atuação nos cuidados de pacientes com AVC, há ampliação do seu conhecimento em relação às diversas necessidades deste tipo de paciente, o que propicia aprimoramento para sua atuação profissional específica. Deve-se enaltecer a necessidade de encaminhamento aos pacientes acometidos pelas doenças neurovasculares aos cuidados dos diferentes profissionais da saúde que compõe a equipe de assistência (médico neurologista, fisioterapeuta, terapeuta ocupacional, enfermeiro, fonoaudiólogo, nutricionista, psicólogo, assistente social e outros) $)^{5}$.

As unidades de recuperação de AVC devem promover reuniões formais entre a equipe interdisciplinar e os pacientes, com o objetivo de identificar e apresentar os problemas para definir, juntamente com os doentes e familiares os objetivos a serem alcançados a curto e em longo prazo. O número total de camas deve ser calculado pela idade, sexo, tipo de incidente e população atendida no hospital. Ainda discute-se questões sobre o atendimento domiciliar, que deve envolver orientações aos profissionais, pacientes e familiares sobre a doença, prevenção e serviços de suporte. Um programa de treinamento para equipe em unidades de AVC é extremamente 
importante, sendo necessário abordar temas como: atuação coordenada de uma equipe interdisciplinar, integração do paciente e familiares com todos os membros da equipe, inclusive em atendimentos domiciliares ${ }^{5}$.

A realização de novos cursos de educação continuada, incluindo apostilas contendo resumos das principais diretrizes (guidelines) para o manejo de pacientes com AVC parece ser emergente e, que será bem aceito pelos diferentes profissionais da saúde nas cidades de São Paulo e Grande ABC.

\section{CONCLUSÃO}

Esta pesquisa de campo nos permite concluir que a utilização de diretrizes (guidelines) para conduzir as condutas assistenciais específicas não é prática usual para maioria dos profissionais da saúde (enfermeiro, fisioterapeuta, assistente social, médico, fonoaudiólogo, terapeuta ocupacional, nutricionista e psicólogo) entrevistados, atuantes nos municípios de São Paulo, Santo André, São Bernardo do Campo e São Caetano do Sul, e que estes possuem muitas e variadas dificuldades e dúvidas quanto as suas atuações profissionais nos cuidados dos pacientes com AVC.

A maioria concorda com a importância e a necessidade e, mostrou interesse em receber orientações por meio de informação (materiais didáticos eletrônicos ou impressos) e formação (cursos de educação continuada) profissional especializada para poderem prestar melhor assistência a esta determinada população, mesmo já as tendo recebido ou as tendo ministrado.

\section{REFERÊNCIAS BIBLIOGRÁFICAS}

1. Lira MMTA, Drumond Jr. M. Estudos Epidemiológicos. Ministério da Saúde - FUNASA (Fundação Nacional de Saúde); 2000.

2. Sociedade Brasileira de Doenças Cerebrovasculares. Primeiro Consenso Brasileiro do tratamento da fase aguda do acidente vascular cerebral. Arq Neuropsiquiatria. 2001; 59:972-980.

3. Thorvaldsen P, Asplund K, Kuulasmaa K, Rajakangas A-M, Schroll M. Stroke incidence, case fatality, and mortality in the WHO MONICA Project. Stroke 1995; 26(3):361-367.

4. Baxter D. Clinical syndromes associated with stroke. In: Brandstater ME, Basmajian JV. Stroke Rehabilitation; 1987. p. 36-54.

5. The European Stroke Initiative Executive Committee and EUSI Writing Committee. European Stroke Initiative Recommendations for Stroke Management - Update 2003. Cerebrovasc Dis 2003; 16:311-337.

6. Sackett DL, Straus SE, Richardson WS, Rosenberg WS, Haynes RB. Evidence-based medicine: How to practice and teach EBM. 2nd. ed. New York: Churchill Livingstone; 2000.

7. Langhorn P, Dennis M. Stroke Units: an evidence based approch. BMJ Books; 1998.

8. Adams Jr HP, Adams RJ, Brott T, del Zoppo GJ, Furlan A, Goldstein LB, Gurbb RL, Higashida R, Kidwell C, Kwiatkowski TG, Marler JR, Hademenos GJ (Stroke Council of the American Stroke Association). Guidelines for the early management of patients with ischemic stroke: a scientific statement from the stroke Council of the American Stroke Association. Stroke 2003; 34:1056-1083.

9. Zwarenstein M, Reeves S, Barr H, Hammick M, koppel L, Athinks J. Interprofissional education: effects on professional practice and health care outcomes (Cochrane Review). In: Cochrane Library, Issue 4, 2003. Chichester, UK: John Wiley \& Sons, Ltd.

10. Feinberg W, Albers G, Barnett H, Biller J, Caplan L, Carter L et al. Guidelines for the management of transient ischemic attacks - from the Ad Hoc Committee on Guidelines for the Management of Transient Ischemic Attacks of the Stroke Council of the American Heart Association. Circulation 1994; 89:2950-65.
11. Adams Jr HP, Brott TG, Crowell RM, Furlan AJ, Gomez CR, Grotta J, Helgason CM, Marler JR, Woolson RF, Zivin JA, el al. Guidelines for the management of patients with acute ischemic stroke. A statement for healthcare professionals from a special writing group of the Stroke Council American Heart Association. Stroke 1994; 25:1901-1914.

12. Aboderin I, Venables G (for Pan European Consensus Meeting on Stroke Management). Stroke management in Europe. J Intern Med 1996;240:173-180.

13. Duncan PW. Synthesis of intervention trials to improve motor recovery following stroke. Top Stroke Rehabil 1997; 3:1-20.

14. European Stroke Initiative. European Stroke Initiative Recommendations for stroke management. Cerebrovasc Dis 2000; 10:335-351.

15. Bhatt DL, Kapadia SR, Yadav JS, Topol EJ. Up-date on clinical trials of antiplatelet therapy for cerebrovascular diseases. Cerebrovasc Dis 2000: 10(suppl 5):34-40.

16. Langhorne P, Pollock A (for the Stroke Unit Trialists' Collaboration). What are the components of effective stroke unit care? Age Ageing 2002: 31:365-371.

17. Brainin M, Steiner M. Acute stroke units in Austria are being set up on a national level following evidence-based recommendations and structural quality criteria. Cerebrovasc Dis 2003; 15(suppl 1):29-32.

18. Duncan PW, Horner RD, Recker DM, Samsa GP, Hoening H, Hamilton B, Laclair BJ, Dudley T. Adherence to Postacute Rehabilitation Guidelines is Associated With Functional Recovery in Stroke. Stroke 2002; 33:167 - 178.

19. Sociedade Brasileira de Doenças Cerebrovasculares. Primeiro Consenso Brasileiro para trombólise no Acidente Vascular Cerebral Isquêmico. Arq Neuropsiquiatr 2002;60:675-680

20. Carson AJ, Ringbauer B, MacKenzie L, Warlow C, Sharpe M. Neurological disease, emotional disorder, and disability: they are related - a study of 300 consecutive new referrals to a neurology outpatient department. J Neurol Neurosurg Psychiatry 2000;68:202-206.

21. Tognola WA. Qualidade de atendimento após acidente vascular cerebral. In: Gagliardi RJ. Doenças Cerebrovasculares: Condutas. São Paulo: Gelgr; 1996. 